\title{
Vom Ende der Natur als normativer Begründungsressource in spätmodernen Gesellschaften
}

\section{Bernhard Gill}

Allgemein wird in der Soziologie heute die selbstkritische Diagnose eines 'ökologischen Defizits' gestellt. Häufig verbunden ist damit die Forderung, 'Natur' als Kategorie in die Soziologie einzuführen. Ich will im folgenden 'Natur' im Hinblick auf ihre Eignung als normative gesellschaftliche Begründungsressource betrachten. Solange man Natur als gegeben und von gesellschaftlicher Manipulation unbeeinflußt konzipieren konnte, eignete sich der Rekurs auf Natur zur stabilen Grenzziehung für die Festlegung von gesellschaftlichen Rechten und Pflichten. Die Grenzziehung zur Natur entschied also einerseits darüber, wer über Beteiligungsrechte verfügen und damit als Rechtssubjekt zur Gesellschaft gehören sollte. Andererseits wurde mit dieser Grenzziehung auch der Geltungsbereich von Pflichten festgelegt: Gesellschaftliche Entscheidungen sind $\mathrm{zu}$ verantworten, naturbedingte Ereignisse sind als Schicksal hinzunehmen.

Neuerdings wird sich die Gesellschaft aber bewußt, daß sie einerseits die Grenze ihres Dominanzbereichs immer weiter ausdehnen kann, zugleich aber mit verstärkten Rückwirkungen aus der Natur in Form technisch vermittelter Sachzwänge und nicht-intendierter Nebenfolgen zu rechnen hat. Die alte Grenzziehung wird also zunehmend problematisch.

1. Der Konflikt um die gesellschaftliche Einbeziehung verschiedener menschlicher Naturen

Neuere Emanzipationsbewegungen - vor allem der Frauen, aber auch von Schwulen, Farbigen, Behinderten, Alten und Kindern - kämpfen gegen gesellschaftliche Marginalisierung und Ausgrenzung, die sich an biologischen Merkmalen festgemacht hat. Sie wenden sich gegen übertriebene Zuschreibungen von Andersartigkeit und versuchen auf den verschiedensten Felder zu beweisen, daß sie im Rahmen eines an den Fähigkeiten weißer Männer orientierten Leistungsideals sehr wohl konkurrenzfähig sind. Sie verlangen zugleich von der Gesellschaft, daß ihnen diejenigen technischen und organisatorischen Hilfestellungen gewährt werden, die zur Kompensation biologisch bedingter Einschränkungen erforderlich sind.

Zunächst wird die moderne, auf der Abgrenzung zur Natur beruhende Gesellschaftsauffassung durch diese Vorstöße nicht bedroht. Die Inklusion bisher margi- 
nalisierter oder ausgegrenzter Menschen liegt im moralischen Horizont der Moderne. Sie stellt prinzipiell kein Problem dar, sofern die neu aufzunehmenden Gesellschaftsmitglieder und ihre Interessen im gesellschaftlichen Binnenverkehr repräsentiert werden können. Was die Subjekte als gesellschaftlich ausweist, ist gerade ihre - in der Cartesianischen Scheidung zwischen res cogitans und res extensa unterstellte - Unabhängigkeit von der Natur.

Schwierigkeiten entstehen allerdings, wenn und soweit die neuen Gesellschaftmitglieder ihre Natur nicht soweit neutralisieren können, daß sie im gesellschaftlichen Verkehr im Verhältnis zur männlichen weißen 'Normalnatur' nicht mehr auffällig wird. Allerdings sind viele der quasi-ImmigrantInnen auch nicht willens, sich in dieser Form zu assimilieren. Sie halten trotz der Integrationsbemühungen an ihrer Differenz fest, kritisieren die dominierende Denkweise und das Leistungsideal und verlangen von der Gesellschaft die Anerkennung und Berücksichtigung ihrer eigenen Denkstile und Handlungsformen. Zumindest in letztgenannter Stoßrichtung wird die Cartesianische Spaltung zwischen Geist und Körper, die die moderne Trennung von Gesellschaft und Natur begründet, tendenziell aufgehoben. In diesem Moment kann auch die schon je gegebene Naturbasiertheit der bis dato dominierenden Verkehrsformen, also die männliche weiße 'Normalnatur', wieder der Wahrnehmung exponiert werden, weil sie nun nicht mehr selbstverständlich ist. Entsprechende Konsequenzen zieht auch die feministische Wissenschafts- und Rationalitätskritik.

Wenn nun die anderen Denkstile und Rationalitäten mit einer abweichenden Natur begründet und zugleich als legitim reklamiert werden, türmt sich im universalistischen Selbstverständnis moderner Gesellschaften allerdings eine Integrationsbarriere auf. Wenn divergierende Denkstile und Rationalitäten aus einer langen Geschichte der fälschlich auf biologische Unterschiede rekurrierenden Fremd- und Selbstzuschreibung resultieren, dann kann nur die langwierige Aufarbeitung dieser Geschichte, wenn nicht zur Konvergenz, so zumindest zur hermeneutischen Verständigung zwischen den biologisch je Anderen beitragen.

Dabei steht die soziale Inklusion, wie oben schon angedeutet, auch in enger Wechselwirkung mit der Proliferation der Technik. Für die Zukunft werden von der Prothetik, der Chirurgie und der Gentechnik weitere Mittel zur Umgestaltung von Menschen nach ihren individuellen Wunschbildern avisiert. Diese Entwicklung kann man als technische Denaturalisierung begreifen, die als Ergänzung oder als Ersatz zur eben beschriebenen sozialen Denaturalisierung auftreten kann.

Die Inklusion wirft aber auch ein Kapazitätsproblem auf. Denn die Ausdehnung von Abwehr- und Anspruchsrechten auf ehemals 'tierähnlich' gehaltene Wesen ist mit einer Beschneidung angestammter Privilegien verbunden: Man darf diese We- 
sen nun nicht mehr als Ressource benutzen und muß überdies die übrigen Ressourcen mit ihnen teilen. Diesem Problem kann nur durch eine - allerdings unwahrscheinliche - Selbstbeschränkung der bereits Etablierten oder durch Produktivitätssteigerungen, also mit (sozial)technischen Mitteln, begegnet werden. Soweit Produktivitätssteigerungen aktuell nicht möglich sind, ergibt sich dann situativ auch immer wieder der Bedarf, Kapazitätsengpässen mit naturalisierenden Ausgrenzungen zu begegnen.

2. Der Konflikt um ehemals als 'natürlich' aufgefaßte Gefahren

Wie eingangs schon angedeutet, entscheidet die normative Grenzziehung zwischen Gesellschaft und Natur auch darüber, was als gleichsam schicksalhafte Gefahr zu akzeptieren und was als entscheidungsbedingtes Risiko zu verantworten ist. Diese Annahme wird auch vom hergebrachten Rechtsdenken gestützt, das zwischen Absicht, grober und einfacher Fahrlässigkeit und unterlassener Hilfeleistung unterscheidet und mit entsprechend von Stufe zu Stufe jeweils abgeschwächten Sanktionen reagiert.

Auch hier sind Grenzerweiterungen als auch Grenzziehungsprobleme zu beobachten, wie ich am Beispiel technisch induzierter Risiken zeigen möchte. Daß sich nicht-intendierte Technikfolgen heute immer schwerer aus dem Gesichtskreis gesellschaftlicher Wahrnehmung verdrängen lassen, hat meines Erachtens drei Gründe:

- $\quad$ Steigendes Ausmaß der Industrialisierung und gewachsenes Zerstörungspotential neuer Techniken.

- Verbesserte Wahrnehmungsinstrumente für ökologische Nebenfolgen von Industrialisierung und Technologisierung.

- Soziologisch besonders interessant für die Entwicklung der ökologischen Frage ist der dritte Gesichtspunkt, der an meine vorigen Ausführungen anknüpft: Durch die erweiterte soziale Inklusion - einerseits innerhalb nationaler Demokratien und andererseits im Rahmen weltgesellschaftlicher Globalisierung - werden die von unerwünschten Technikfolgen Betroffenen konfliktfähiger. Ehemals sozial und räumlich ungesehen und ungestraft externalisierbare Folgen werden nun erkannt und zugerechnet.

Dagegen wird die Unterscheidung zwischen Technik und Natur von Technologiebefürwortern eingesetzt, um befürchtete oder eingetretene Schäden ursächlich auf Natur zuzurechnen. Bei der Gentechnik behaupten deren Befürworter, daß die Gentechnik nichts anderes mache als die 'Natur'. Der gleichen Logik folgen Kritiker, wenn sie für den Verzicht auf eine in ihren Augen 'unnatürliche' Technologie 
plädieren oder für die Beibehaltung von herkömmlichen, in ihren Augen 'naturnahen' Technologien eintreten. Die 'Natur' dient in beiden Argumentationsstragien als Entlastungskategorie, um die Zuschreibung von Verantwortung abzuwehren, sei es, indem man die Technikfolgen oder die Technologien selbst naturalisiert.

Das herkömmliche Rechtsdenken, auf das beide Seite hier rekurrieren, reflektiert noch eine Situation, in der menschliche Handlung in ihrer Wirkung überschaubar, die Natur dagegen übermächtig schien. Moderne Risikokonflikte zeichnen sich jedoch dadurch aus, daß untereinander vernetzte Akteure Technologien in Gang setzen, die sich in ihrer Wirkung - jedenfalls in wissenschaftlicher, d.h. systematisch erweiterter Wahrnehmung - mit Natureffekten vielfach überlagern. Daher ist häufig weder genau festzustellen, wer nun eigentlich entschieden hat, noch ob es sich bei den befürchteteten oder eingetretenen Folgen um einen technisch induzierten Schaden oder ein natürliches Phänomen handelt. Das Kausalitätsprinzip wird also zunehmend vom Finalitätsprinzip der Verantwortung überlagert: Es zählt weniger, wer 'Schuld hat', sondern was zu tun ist, um dem Schaden vorzubeugen oder ihn zu beheben.

\section{Entwicklungsperspektiven der hier aufgezeigten Grenzziehungskonflikte}

Wenn also im Endeffekt die Unterscheidung zwischen Technik und Natur in Risikokonflikten unter aufgeklärten Diskursbedingungen weitgehend obsolet wird, dann geht es schließlich nur noch um die Frage, wie hoch jeweils die bekannten und die bisher noch unbekannte Risiken einzuschätzen sind. Es wird dann kein prinzipieller Unterschied mehr gemacht, ob Risiken von der Gesellschaft oder der Natur herrühren, oder die Gesellschaft oder die Natur betreffen. Die Unterscheidung zwischen Gesellschaft und Natur wird also in die Unterscheidung zwischen Wissen und Nicht-Wissen transformiert. Diese Unterscheidung fällt, zumindest nach herkömmlicher Auffassung, in den Kompetenzbereich des Wissenschaftssystems. Die anderen Subsysteme werden also in ihrer Funktionsweise, soweit sie bisher auf die Unterscheidung von Gesellschaft und Natur und nun auf die Unterscheidung von Wissen und Ungewißheit gründet, in besonderer Weise vom Wissenschaftssystem abhängig.

Die Natur verliert dabei auf der symbolischen Ebene der Ordnungsbegriffe ihre moralische Funktion als Entlastungskategorie und wird durch das funktionale Äquivalent der Ungewißheit abgelöst. Bekannte Risiken können nun nicht mehr (so leicht) in ihrer Wirkung auf sozial Schwächere, entferntere Territorien oder Naturräume abgewälzt werden, soweit diese sozial inkludiert bzw. als wertvolle Ressource vergesellschaftet werden. Nur bisher noch unbekannte Risiken sind verant- 
wortungsentlastet und werden, zum Teil auch durch neue Technologien, mit denen die bekannten Risiken der alten Technologien vermieden werden, auf kommende Generationen übertragen. Das scheint der trigonometrische Punkt zu sein, auf den modernes Denken in seiner Zuspitzung auf ökologische Modernisierung zwangsläufig hinzutreiben scheint.

Allerdings gründet das Wissenschaftssystem nun selbst wieder auf der zunehmend erodierenden Grenzziehung zwischen Gesellschaft und Natur, die ihre 'Objektivität' ehemals sicherstellte. Mehr noch: Indem der Stand des Wissens überhaupt thematisiert wird, wird zugleich auch deutlich, daß sich jenseits des erreichten Standes die weite Sphäre des Noch-Nicht-Wissens öffnet. Das sogenannte Restrisiko, das aus der Perspektive des Stands der Wissenschaft als äußerst gering erscheint, wird aus der Perspektive des Noch-Nicht-Wissen-Könnens zum Zukunftsrisiko schlechthin. Entsprechende Erfahrungen mit dem Noch-Nicht-WissenKönnen der Vergangenheit sind uns heute allgegenwärtig. Entscheidungen unter Ungewißheit aber können legitimerweise dann nur noch von allen, die gleich viel oder gleich wenig wissen, getroffen werden.

Hier wird wieder die Inklusionsproblematik virulent, die ich in Punkt 1 angesprochen habe. Es geht dann um die Frage, wer zu beteiligen ist und welche Rationalitätsformen berücksichtigt werden sollen, wenn der durch funktionale Differenzierung ohnehin schon zersplitterte Universalitätsanspruch der euro- und androzentrischen 'Normalvernunft' aufgegeben werden müßte. Ausgrenzungen ließen sich mit dem Rekurs auf Natur kaum mehr rechtfertigen. Die Grenze zwischen Natur und Gesellschaft, die ehedem a priori festlegte, wer zu beteiligen und was zu verantworten sei, würde vielmehr selbst zum Verhandlungsgegenstand. Legitimation wäre dann nur noch durch permanente gesellschaftliche Beteiligung und Aushandlung sicherzustellen, wobei auch die Verfahren selbst immer wieder zur Disposition stünden.

Das scheint der trigonometrische Punkt zu sein, auf den reflexive Modernisierung hinauslaufen könnte. Die große Frage bleibt hier aber, wie dieser nur im Sinne kleinräumiger und zeitaufwendiger Demokratie vorstellbare Prozess den im Zeichen der Globalisierung in immer kürzerer Folge anstehenden Entscheidungen mit immer größerer Tragweite gewachsen sein soll. In diesem Kontext scheint die Perspektive ökologischer Modernisierung aufgrund der besser eingespielten Bezüge zu den dominierenden Institutionen Zeitgewinne zu verschaffen und die kurzfristigen Transaktionskosten zu reduzieren. Insofern ist schwer abzusehen, welche Entwicklungsperspektive sich schließlich durchsetzen wird. 